

\title{
DISEÑO DE EDIFICIOS DE OFICINAS SUSTENTABLES PARA PROMOVER OCUPANTES SUSTENTABLES
}

DESIGN OF GREEN OFFICE BUILDINGS TO PROMOTE GREEN OCCUPANTS

\author{
FERNANDO CHÁVEZ-FINOL \\ Arquitecto \\ Estudiante del Doctorado en Arquitectura y Urbanismo \\ Universidad del Bío-Bío \\ Concepción, Chile \\ https://orcid.org/0000-0002-4889-9305 \\ fernando.chavez1801@alumnos.ubiobio.cl
}

\author{
MAUREEN TREBILCOCK-KELLY \\ Doctora en Arquitectura Sustentable \\ Académica jornada completa del Departamento de Diseño y \\ Teoría de la Arquitectura, Universidad del Bío-Bío \\ Concepción, Chile \\ https://orcid.org/0000-0002-1984-0259 \\ mtrebilc@ubiobio.cl
}

\author{
MARÍA BEATRIZ PIDERIT-MORENO \\ Doctora en Arquitectura y Clima \\ Académica jornada completa del Departamento de Diseño y \\ Teoría de la Arquitectura, Universidad del Bío-Bío \\ Concepción, Chile \\ https://orcid.org/0000-0001-9733-6608 \\ mpiderit@ubiobio.cl
}

\begin{abstract}
RESUMEN
Escasos son los trabajos que se enfocan en investigar el potencial de los edificios sustentables de promover sustentabilidad en sus ocupantes. Por ello, el siguiente estudio tiene como objetivo analizar el uso de créditos LEED para fomentar comportamientos pro-ambientales. La metodología utilizada es de carácter exploratoria y de lógica descriptiva, y analiza comparativamente edificios de oficinas certificados LEED [Argentina $(n=351)$, Chile $(n=494)$, Colombia $(n=432)$, Perú $(n=282)$ ] en el período 2012-2020. Los resultados revelaron que los créditos más empleados fueron: "Acceso a Transporte

Público" (99,34\%), "Densidad del Entorno" (98,34\%) y "Pautas de diseño y construcción para inquilinos" (96,53\%); y los menos empleados: "Puesta en servicio mejorada" (44,30\%), "Luz diurna"

$(31,31 \%)$ y "Controlabilidad de los sistemas" (7,53\%). Se concluye, finalmente, que aquellos que optan por incluir al ocupante en el diseño, eligen intervenir en la cultura, mientras que quienes optan por no hacerlo, eligen la tecnología.
\end{abstract}

Palabras clave

diseño sustentable, edificios de oficinas, sistemas de certificación en la sustentabilidad, comportamiento pro-ambiental.

\footnotetext{
Few studies focus on researching the potential of sustainable buildings to promote the sustainability of their occupants. Therefore, this study aims at analyzing the use of LEED credits, with the intention of promoting pro-environmental behaviors. The methodology is exploratory in nature, with a descriptive logic, and comparatively analyzes LEED-certified office buildings [Argentina $(n=351)$; Chile $(n=494)$; Colombia ( $n=432)$; and Peru $(n=282)]$, between 2012 and 2020. The results revealed that the most used credits were: "Access to Public Transportation", (99.34\%); "Surrounding Density"; (98.34\%); and,

"Tenant construction and design guidelines", (96.53\%); and the least used ones were: "Enhanced commissioning", (44.30\%); "Daylight" (31.31\%); and, "Controllability of systems", (7.53\%). It is concluded that those who choose to include the occupant in the design, choose to intervene in the culture, while those who choose not to include them, choose technology.
} 


\section{INTRODUCCIÓN}

A principios de los años noventas, una de las propuestas más aceptadas para avanzar en el cumplimiento de los desafíos del desarrollo sustentable fue la creación de sistemas de evaluación, calificación y certificación en la sustentabilidad de los edificios en sus etapas de diseño, construcción, operación y mantenimiento (Chwieduk, 2003; R. Cole, 1999; 2002; Ding, 2008). Estos métodos surgieron con el fin de certificar la sustentabilidad de edificios a través de indicadores consensuados y medibles, que proporcionaron, además, procesos y pautas prácticas para diseñar y evaluar el rendimiento del edificio a través de una lista de verificación fácil de usar (Gou, 2016). Tales sistemas de certificación en la sustentabilidad promueven edificios de bajo impacto ambiental (Chwieduk, 2003; R. Cole, 1999; Ding, 2008), pero la realidad ha demostrado que esto depende en gran medida de los comportamientos de los ocupantes y que se ve frecuentemente desvirtuado por errores en la construcción, ajustes incorrectos de los equipos y la simplificación excesiva en los modelos de simulación (van den Brom, Meijer y Visscher, 2016; Fabi, Andersen y Corgnati, 2011). Varios son los estudios que han analizado la brecha entre los desempeños esperados del edificio y los desempeños reales influidos por factores humanos ( $D^{\prime} O c a$, Hong y Langevin, 2018; Hong, Yan, D'Oca, y Chen, 2017; Stazi y Naspi, 2018), pero son pocos los estudios que han abordado el efecto del diseño sustentable en la conciencia, actitudes, valores y conocimiento medioambiental, como predictores de comportamientos pro-ambientales de los ocupantes (Mokhtar y Wilkinson, 2015; Deuble y de Dear, 2012; 2009; Kirk, 2010; McCunn y Gifford, 2012; Rashid, Spreckelmeyer y Angrisan, 2012).

Para esta investigación, se define al "Edificio sustentable" como aquel diseñado para ser acreditado con un sistema de certificación en la sustentabilidad, validado por un tercero. El sistema más conocido internacionalmente es LEED, Liderazgo en Energía y Diseño Ambiental (Leadership in Energy and Environmental Design), desarrollado en los Estados Unidos en 1998. LEED está elaborado para clasificar todo tipo de edificaciones en base a principios consensuados en materia energética y ambiental, e intenta lograr un equilibrio entre las prácticas conocidas y establecidas con conceptos emergentes. Está organizado principalmente en 5 categorías de evaluación: "Sitios sustentables"; "Eficiencia del agua"; "Energía y atmósfera"; "Materiales y recursos"; y, "Calidad ambiental interior". En cada una de sus categorías, los proyectos deben satisfacer determinados "Prerrequisitos" y ganar puntos o créditos. Los "Prerrequisitos" establecen los requisitos mínimos que todos los edificios deben cumplir para lograr la certificación LEED, y los créditos, los que realmente distinguen al edificio. Además de LEED, existen otros métodos con impacto global, tales como BREEAM, HQE, Passivhaus, etc. (Mattinzioli, Sol-Sánchez, Moreno, Alegre y Martínez, 2020). Cada país ha avanzado en el desarrollo de su propio sistema con el fin de incluir criterios locales, tales como Green Rating for Integrated Habitat Assessment (GRIHA), en la India, Green Building Rating System (SAGRS), en Arabia Saudita, y la Certificación de Edificio Sustentable (CES), en Chile, entre otros (Ahmed, Abul Hasan y Mallick, 2016). La importancia de estos sistemas para el diseño arquitectónico debería ser un aspecto a destacar, ya que influyen en la forma en que los diseñadores piensan y concretan sus proyectos (Labartino, 2018). Debido a las necesidades y requerimientos de los ocupantes, la mayoría de los sistemas apunta a un balance entre la sustentabilidad ambiental y la sustentabilidad social (Moezzi, 2009). En esa línea, Heerwagen (2000) manifiesta que los beneficios de los edificios sustentables se dan cuando el edificio y sus ocupantes se tratan como un sistema integrado, y Lee (2010) argumenta que los edificios sustentables tienen mejores posibilidades de éxito si a los ocupantes se les instruye en la motivación sustentable y en los principios de la organización detrás de la implementación del sistema de certificación en la sustentabilidad. Diversos son los estudios comparativos entre edificios sustentables y convencionales que buscan conocer el potencial de los primeros de promover sustentabilidad en los ocupantes. Khashe et al. (2015) concluyeron que el conocimiento de los ocupantes del edificio sobre los problemas medioambientales podría mejorar sus comportamientos en pro del ambiente. El trabajo de Steinberg, Patchan, Schunn y Landis., (2009) advirtió que un grupo de ocupantes que iba a ser trasladado a un edificio sustentable declaró mayor disposición a cambiar sus comportamientos, que aquellos ocupantes que iban a mantenerse en el edificio convencional. Mokhtar, Wilkinson y Fassman (2015), por su parte, patentizaron que los ocupantes de los edificios sustentables adoptaban más cambios en sus comportamientos que los ocupantes de los edificios convencionales, debido a las estrategias de intervención implementadas por la organización en los edificios sustentables. Hill et al. (2019) exploraron otros factores en los ocupantes, como el de la conciencia medioambiental, las percepciones y la facilidad o dificultad percibida de los comportamientos, para concluir que estar en un edificio sustentable influye en los ocupantes en términos de mostrar un comportamiento pro-ambiental. Y Tezel y Giritli (2019) hallaron que el valor, la creencia y la conciencia medioambiental eran, estadísticamente, predictores del comportamiento pro-ambiental en el lugar de trabajo, y que los ocupantes de oficinas sustentables mostraban una mayor conciencia sobre las características sustentables de los edificios. Ahora bien, a pesar de constatar una mayor conciencia, los ocupantes de oficinas sustentables revelaron menos evidencia de comportamientos pro-ambientales en comparación con los que trabajaban en edificios de oficinas no sustentables, a partir de lo cual se visibilizó la necesidad de mayores esfuerzos en la formación sobre cuestiones de sustentabilidad en la sociedad.

En contraposición, otros estudios han demostrado que los edificios sustentables no promueven, en general, comportamientos pro-ambientales en los ocupantes, en comparación con edificios convencionales o no sustentables. Por ejemplo, Hostetler y Noiseux (2010) concluyeron que 
los nuevos residentes no demuestran el conocimiento, las actitudes y los comportamientos pro-ambientales para hacer que las comunidades sustentables funcionen con los objetivos de sustentabilidad proyectadas en el diseño. De acuerdo con McCunn y Gifford (2012), ni el compromiso ni las actitudes medioambientales se correlacionan con los atributos sustentables de los edificios. Según Rashid et al. (2012), no existe evidencia de efectos directos de los atributos arquitectónicos de un edificio sustentable en la conciencia medioambiental y la imagen organizacional de los ocupantes. En ese sentido, hay investigaciones que profundizan en algunas razones; una de ellas mostró que cuando la opción de reciclaje estaba disponible, las personas aumentaban el uso de productos que eran gratuitos (papel de oficina, toallas de papel de baño, etc.), creando efectos adversos en la sustentabilidad (Catlin y Wang, 2013). Este tipo de comportamiento podría explicarse por el hecho de que un edificio sustentable puede ser considerado una especie de compensación para aliviar las emociones negativas, como la culpa asociada con el comportamiento derrochador (Bamberg y Möser, 2007).

La sustentabilidad de un edificio también puede parecer una rectificación, que desmejora las percepciones de riesgo de los ocupantes y aumenta sus intenciones de usar más energía (Bolton, Cohen y Bloom, 2006). En otras palabras, ocupar un edificio sustentable podría considerarse una compensación para los ocupantes y otorgarles una licencia para un comportamiento menos ambiental, ya que pueden percibir y tener la sensación de que las estrategias de la sustentabilidad presentes en el edificio compensan su comportamiento poco amigable con el medioambiente. Bajo esta premisa, ciertos autores proponen la noción de "Diseño robusto" (Robust design) (Buso, Fabi, Andersen y Corgnati, 2015; Karjalainen, 2016; O'brien, 2013; Palme, Isalgue, Coch-Roura, Serra y Coch, 2006), fundamentada en que "los ocupantes no comprenden los principios de funcionamiento de los edificios y que utilizan los sistemas de forma no óptima o, incluso, inapropiada desde el punto de vista energético" (Karjalainen, 2016, p. 1.257).

La expresión "Ocupante sustentable" (OS) aparece por primera vez en el trabajo de Browne y Frame (1999), en donde concluyen que "edificios sustentables necesitan ocupantes sustentables" (green buildings need green occupants), partiendo de la base de que la tecnología por sí misma no es suficiente para lograr los objetivos de sustentabilidad propuestos en el diseño de los edificios, y que se debería incluir al ocupante en el proceso. Luego, dicha concepción fue tomada en cuenta en otras investigaciones (Deuble, 2007; Deuble y de Dear, 2012; 2009; Wu, 2016; Wu, Green, Chen, Tang y Yang, 2015; Wu, Greaves, Chen y Grady, 2017; Wu, Kim, et al., 2017). Deuble (2007) profundizó en la noción de OS para terminar definiendo a este ocupante como aquel que entiende las estrategias sustentables presentes en el edificio y que, a su vez, posee alto nivel de conciencia medioambiental; aspectos medibles con la "Escala del Nuevo Paradigma Ecológico" (NEP-R) (Dunlap, Van Liere, Mertig y Jones, 2000; Dunlap y Van Liere, 1978).
Sin embargo, algunos autores descubren ocupantes "grises" en edificios sustentables debido al "Efecto rebote" (Rebound effect) (Catlin y Wang, 2013; Frondel, 2004; Sorrell, 2007). En esa misma lógica, se podría argumentar que ocupantes con altos niveles de conciencia medioambiental de edificios no sustentables, podrían compensar la ausencia de estrategias sustentables en el edificio y comportarse, por ello, en pro del medioambiente. A partir de este marco, aquí se concebirá al OS como al ocupante con comportamientos pro-ambientales (CPA) en el edificio y, paralelamente, al "comportamiento pro-ambiental", como uno "que conscientemente busca minimizar el impacto negativo de las acciones de uno en el mundo natural y construido (por ejemplo, minimizar el consumo de recursos y energía, el uso de sustancias no tóxicas, reducir la producción de desechos)" (Kollmuss y Agyeman, 2002, p. 240).

Otros trabajos se abocan al uso del edificio sustentable como promotor de la sustentabilidad, a través del concepto "Edificio Verde Educador" (Teaching Green Building) (L. Cole, 2014; 2018; L. Cole y Hamilton, 2019), el cual ejemplifican con el caso del sistema de certificación LEED que ofrece un crédito para los proyectistas que emplean al edificio certificado de la escuela (green school building) como herramienta de enseñanza (L. Cole, 2013). Asimismo, se ha estudiado la "comunicación de la sustentabilidad" por medio de los atributos arquitectónicos presentes en el edificio (Cranz, Lindsay, Morhayim y Lin, 2014; Wu, 2016; Wu et al., 2015; Wu, Greaves, et al., 2017; Wu, Kim, et al., 2017), para concluir que se debe continuar promoviendo el uso de la señalización educativa en el diseño de edificios sustentables -medida que resultó ser, en uno de los casos (Wu, Kim et al., 2017), el comunicador más eficaz de la sustentabilidad-, como también alentar más análisis sobre el uso innovador del diseño de edificios sustentables, como comunicadores eficaces para promover educación sobre la sustentabilidad entre ocupantes de edificios.

Conforme a lo descrito, no es suficiente lo que se ha investigado en la relación del diseño de edificios sustentables y su efecto en el fomento de la sustentabilidad en los ocupantes. Por lo tanto, el objetivo general de este trabajo es explorar la relación del edificio sustentable con el ocupante, a través del análisis, identificación y clasificación de los criterios asociados, con la intención de promover CPA en dichos ocupantes, mediante un sistema de certificación en la sustentabilidad, en base a un modelo teórico -lo que corresponde al objetivo específico 1 (OE1). Consiguientemente, se lleva a cabo un análisis comparativo en edificios de oficinas certificados LEED en Argentina, Chile, Colombia y Perú, entre los años 2012 y 2020 -lo que corresponde al objetivo específico 2 (OE2).

\section{METODOLOGÍA}

La investigación aquí expuesta tiene un carácter exploratorio (Hernández Sampieri, Fernández y del Pilar, 2010), pues significa uno de los pocos acercamientos al fenómeno de 
las estrategias de diseño en un edificio sustentable para promover sustentabilidad (concretamente en CPA) en los ocupantes. Las estrategias de diseño son representadas en los requerimientos (créditos) LEED. El método de selección de los casos es no probablístico y los tipos de muestras (sistemas y países), de tipo "guiadas por uno o varios propósitos" (Hernández Sampieri et al., 2010, p. 396). Como este estudio forma parte de una tesis de doctorado en curso en Chile, el criterio de selección del sistema de certificación es el de mayor número de proyectos registrados en el país; la misma lógica se aplica para la selección de la versión del sistema como de la función de los edificios certificados. Con respecto a la selección de los países, se elegirán aquellos con un número similar y abordable de proyectos registrados en Chile, bajo el argumento de "capacidad operativa de recolección" (Hernández Sampieri et al., 2010, p. 402). Como método de validación del análisis, identificación y clasificación de los créditos del sistema de certificación para generar CPA en los ocupantes, se acudió a la evaluación de juicio de expertos (Garrote y Rojas, 2015). En cuanto al uso de los créditos en los edificios certificados en los países, se efectuó un análisis comparativo, a través de la descripción de los promedios de los créditos LEED obtenidos en cada caso/país y de la descrpción de la desviación estándar de los créditos LEED obtenidos en cada caso/país.

Como soporte teórico, a fin de analizar, identificar y clasificar los créditos LEED con potencial de promover CPA en los ocupantes, se correspondieron los créditos LEED con la variable "Posibilidades de actuar pro-ambientalmente", del "Modelo de comportamiento ecológico" (Fietkau y Kessel [1981] cit. en Kollmuss y Agyeman, 2002), en donde la variable es definida como factores externos (infraestructura o económicos) que permiten o dificultan que las personas actúen pro-ambientalmente. Las otras variables del modelo teórico son: "Actitud y valores"; "Incentivos de comportamiento"; "Retroalimentación percibida sobre el comportamiento pro-ambiental"; y, "Conocimiento", -una variable que no influye directamente en el comportamiento, sino que actúa como un modificador de actitudes y de valores-.

La literatura especializada ha identificado la relación entre los factores socio-psicológicos y los CPA mediante la conceptualización teórica o los estudios de casos empíricos, como la teoría del comportamiento planificado (Ajzen, 1991; Harland, Staats y Wilke, 1999), el modelo de activación de normas (NAM) (Lindenberg y Steg, 2007), la teoría del valor-creencia-norma (VBN: value-belief-norm (Stern, 2000), el nuevo paradigma ambiental (o ecológico) (Dunlap et al., 2000; Dunlap y Van Liere, 1978) y la teoría del apego al lugar (Ramkissoon, Weiler y Smith, 2012). Bamberg y Möser (2007) exponen, en este sentido, que el comportamiento pro-ambiental probablemente se ve mejor como una mezcla de interés propio (por ejemplo, para seguir una estrategia que minimice el propio riesgo para la salud) y de interés hacia otras personas, la próxima generación, otras especies o ecosistemas completos (por ejemplo, previniendo la contaminación del aire que puede causar riesgos para la salud de otros y / o el clima global). Esa combinación de intereses propios y motivos prosociales es evidenciada en la formulación del "Modelo de comportamiento ecológico", criterio complementario para la selección del modelo.

En la Figura 1 se grafica el esquema metodológico de la investigación.

\section{MUESTRA:}

OE1: Sistemas de certificación en la sustentabilidad en Chile

OE2: Países con número similiar de proyectos certificados del sistema de certificación en la sustentabilidad seleccionado en OE1

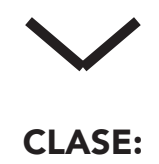

OE1/OE2: No probabilística o dirigida

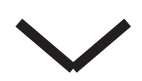

ANÁLISIS:

OE1: Descriptivo

OE2: Descriptivo y comparativo.

Figura 1. Esquema metodológico. Fuente: Elaboración de los autores.

\section{RESULTADOS Y DISCUSIÓN}

\section{RESULTADOS}

El sistema de certificación con mayor número de proyectos registrados en Chile es LEED, con 494 proyectos inscritos (Chile GBC, 2020); los otros sistemas de certificación en el país son: EDGE, Excellence In Design For Greater Efficiencies (Excelencia en el diseño para una mayor eficiencia), WELL, del International WELL Building Institute, y el sistema nacional de Chile, CES, Certificación Edificio Sustentable. Los países con un número similar de proyectos registrados en Chile, en el sistema de certificación LEED, son: Argentina $(n=351)$, Colombia $(n=432)$ y Perú $(n=282)$ (Chile GBC, 2020). Esta información, como la del resto de Latinoamérica, se puede apreciar en la Tabla 1. 


\begin{tabular}{|c|c|}
\hline País & Inscritos \\
\hline Brasil & 1589 \\
\hline México & 1217 \\
\hline Chile & 494 \\
\hline Colombia & 432 \\
\hline Argentina & 351 \\
\hline Perú & 282 \\
\hline Costa Rica & 228 \\
\hline Guatemala & 91 \\
\hline Ecuador & 40 \\
\hline Uruguay & 36 \\
\hline Paraguay & 14 \\
\hline Bolivia & 6 \\
\hline
\end{tabular}

Tabla 1. Proyectos registrados LEED en países de Latinoamérica hasta diciembre 2020. Fuente: Elaboración de los autores, con base en Chile GBC (2020)

\begin{tabular}{|c|c|c|c|c|}
\hline & Argentina & Chile & Colombia & Perú \\
\hline $\begin{array}{c}\text { Edificio de } \\
\text { Oficinas }\end{array}$ & $\begin{array}{c}84 \\
(100,00)\end{array}$ & $\begin{array}{c}121 \\
(100)\end{array}$ & $89(100)$ & $65(100)$ \\
\hline $\begin{array}{c}\text { LEED-CS } 1.0 \\
\text { Pilot }\end{array}$ & $0(0,00)$ & $1(0,83)$ & $0(0,00)$ & $0(0,00)$ \\
\hline LEED-CS 2.0 & $3(3,57)$ & $8(6,61)$ & $1(1,12)$ & $0(0,00)$ \\
\hline LEED-Cl 2.0 & $0(0,00)$ & $0(0,00)$ & $2(2,25)$ & $1(1,54)$ \\
\hline LEED-NC 2.2 & $0(0,00)$ & $2(1,65)$ & $3(3,37)$ & $0(0,00)$ \\
\hline $\begin{array}{c}\text { LEED-CS } \\
\text { v2009 }\end{array}$ & $24(28,57)$ & $\begin{array}{c}69 \\
(57,02)\end{array}$ & $50(56,18)$ & $38(58,46)$ \\
\hline $\begin{array}{c}\text { LEED-NC } \\
\text { v2009 }\end{array}$ & $24(28,57)$ & $\begin{array}{c}23 \\
(19,01)\end{array}$ & $12(13,48)$ & $3(4,62)$ \\
\hline $\begin{array}{c}\text { LEED-Cl } \\
\text { v2009 }\end{array}$ & $15(17,86)$ & $\begin{array}{c}15 \\
(12,40)\end{array}$ & $11(12,36)$ & $7(10,77)$ \\
\hline $\begin{array}{c}\text { LEED-EB:OM } \\
\text { v2009 }\end{array}$ & $13(15,48)$ & $1(0,83)$ & $2(2,25)$ & $7(10,77)$ \\
\hline $\begin{array}{c}\text { LEED v4 } \\
\text { O+M: EB }\end{array}$ & $0(0,00)$ & $1(0,83)$ & $0(0,00)$ & $0(0,00)$ \\
\hline $\begin{array}{c}\text { LEED v4 } \\
\text { BD+C: NC }\end{array}$ & $1(1,19)$ & $0(0,00)$ & $1(1,12)$ & $0(0,00)$ \\
\hline $\begin{array}{c}\text { LEED v4 } \\
\text { ID+C: } \mathrm{Cl}\end{array}$ & $4(4,76)$ & $1(0,83)$ & $6(6,74)$ & $9(13,85)$ \\
\hline $\begin{array}{c}\text { LEED v4.1 } \\
\text { O+M: Interiors }\end{array}$ & $0(0,00)$ & $0(0,00)$ & $1(1,12)$ & $0(0,00)$ \\
\hline
\end{tabular}

Tabla 3. Diferentes versiones LEED de "Edificios de Oficinas". Fuente: Elaboración de los autores. (Las cifras entre paréntesis representan el porcentaje [\%]).

\begin{tabular}{|c|c|c|c|c|}
\hline & Argentina & Chile & Colombia & Perú \\
\hline $\begin{array}{c}\text { TOTAL } \\
\text { Registrados } \\
\text { LEED }\end{array}$ & $\begin{array}{c}348 \\
(100,00)\end{array}$ & $\begin{array}{c}490 \\
(100,00)\end{array}$ & $\begin{array}{c}428 \\
(100,00)\end{array}$ & $\begin{array}{c}281 \\
(100,00)\end{array}$ \\
\hline $\begin{array}{c}\text { NO } \\
\text { Certificados + } \\
\text { Confidenciales }\end{array}$ & $\begin{array}{c}206 \\
(59,20)\end{array}$ & $\begin{array}{c}256 \\
(52,24)\end{array}$ & $\begin{array}{c}270 \\
(63,08)\end{array}$ & $\begin{array}{c}184 \\
(65,48)\end{array}$ \\
\hline $\begin{array}{c}\text { TP: NO } \\
\text { Oficina }\end{array}$ & $58(16,67)$ & $\begin{array}{c}113 \\
(23,06)\end{array}$ & $69(16,12)$ & $32(11,39)$ \\
\hline TP: Oficinas & $84(24,14)$ & $\begin{array}{c}121 \\
(24,69)\end{array}$ & $89(20,79)$ & $65(23,13)$ \\
\hline
\end{tabular}

Tabla 2. Proyectos registrados LEED en Argentina, Chile, Colombia y Perú, clasificados en: "NO Certificados", "Confidenciales" y "Tipo de proyecto (TP)". Fuente: Elaboración de los autores. (Las cifras entre paréntesis representan el porcentaje [\%]).

Se usó el archivo público de la página del U.S. Green Building Council (https://www.usgbc.org/), para filtrar la información y obtener los proyectos certificados. En la Tabla 2 se muestra un resumen de la clasificación realizada de la información por proyectos registrados, filtrados según el contenido: "NO Certificados + Confidenciales", proyectos, a la fecha, registrados como confidenciales y no certificados; "NO Oficina", proyectos certificados y registrados con función/tipo diferentes a Oficinas (Office); $y_{1}$ "Oficinas", proyectos certificados y registrados con función/tipo Oficinas (Office), en el archivo base, en la columna ProjectTypes (Tipo de proyecto).

La versión con mayor número de proyectos registrados fue la versión LEED-CS v2009 "Core and Shell" (v3), por ser la más usada en la base de datos entre el período 2012 2020, como expresa la Tabla 3.

Una vez seleccionada la versión con mayor número de proyectos registrados, se clasificaron los créditos en tres grupos: "Directos", "Indirectos" y "Otros". "Directos", porque en la descripción del "Propósito" (Intent) en el manual, se establece directamente la intencionalidad de promover CPA en los ocupantes, como ocurre, por ejemplo, en los créditos de transporte alternativo: SSc4.1; SSc4.2; SSc4.3; SSc4.4: "Reducir la contaminación y los impactos del desarrollo de la tierra por el uso del automóvil" (To reduce pollution and land development impacts from automobile use). "Indirectos", porque en la descripción del "Propósito" (Intent) no se establece directamente las intencionalidad de promover CPA en los ocupantes, sino en otro lugar de las descripciones del crédito, como en actividades que se solicitan para obtener los puntajes, como por ejemplo, la de elaborar encuestas a los ocupantes, en el caso del crédito "IEQc3: Puesta en servicio mejorada" (Enhanced Commissioning), o en el del crédito "SSc9: Pautas de diseño y construcción para inquilinos" (Tenant design and construction guidelines), en donde se solicita redactar un manual de instrucciones de las estrategias 


\begin{tabular}{|c|c|c|c|c|c|c|}
\hline \multirow{2}{*}{$\begin{array}{c}\text { Categoría Ambiental } \\
\text { LEED }\end{array}$} & \multirow{2}{*}{$\begin{array}{l}\text { Código } \\
\text { LEED }\end{array}$} & \multirow{2}{*}{ Identificación LEED } & \multirow[b]{2}{*}{ Prerrequisito } & \multicolumn{3}{|c|}{$\begin{array}{c}\text { Categoría Crédito - LEED / } \\
\text { Estudio (*) }\end{array}$} \\
\hline & & & & $\begin{array}{l}\text { Directo } \\
(*)\end{array}$ & $\begin{array}{l}\text { Indirecto } \\
(*)\end{array}$ & $\begin{array}{c}\text { Otro } \\
(*)\end{array}$ \\
\hline $\begin{array}{l}\text { Materiales y Recursos } \\
\text { (Materials and Resources) }\end{array}$ & MRp1 & $\begin{array}{c}\text { Almacenamiento y recolección } \\
\text { de reciclables }\end{array}$ & $\checkmark$ & & & \\
\hline \multirow[t]{7}{*}{$\begin{array}{l}\text { Parcelas Sustentables } \\
\text { (Sustainable Sites) }\end{array}$} & $\mathrm{SSc} 2$ & $\begin{array}{c}\text { Densidad del Entorno y Usos } \\
\text { Diversos }\end{array}$ & & $\checkmark$ & & \\
\hline & $\mathrm{SSc} 4.1$ & $\begin{array}{c}\text { Transporte alternativo: acceso al } \\
\text { transporte público }\end{array}$ & & $\checkmark$ & & \\
\hline & $\mathrm{SSc} 4.2$ & $\begin{array}{c}\text { Transporte alternativo: } \\
\text { almacenamiento de bicicletas y } \\
\text { vestuario }\end{array}$ & & $\checkmark$ & & \\
\hline & $\mathrm{SSc} 4.3$ & $\begin{array}{c}\text { Transporte alternativo: vehículos } \\
\text { de bajas emisiones y de bajo } \\
\text { consumo de combustible }\end{array}$ & & $\checkmark$ & & \\
\hline & $\mathrm{SSc} 4.4$ & Huella de aparcamiento reducida & & $\checkmark$ & & \\
\hline & SSc5.2 & $\begin{array}{l}\text { Desarrollo del sitio: maximice el } \\
\text { espacio abierto }\end{array}$ & & & $\checkmark$ & \\
\hline & SSc9 & $\begin{array}{c}\text { Pautas de diseño y construcción } \\
\text { para inquilinos }\end{array}$ & & & $\checkmark$ & \\
\hline $\begin{array}{c}\text { Energía y Atmósfera } \\
\text { (Energy and Atmosphere) }\end{array}$ & EAc3 & Puesta en servicio mejorada & & & $\checkmark$ & \\
\hline \multirow{3}{*}{$\begin{array}{l}\text { Calidad Ambiental Interior } \\
\text { (Indoor Environmental } \\
\text { Quality) }\end{array}$} & IEQc6 & $\begin{array}{c}\text { Controlabilidad de los sistemas - } \\
\text { Confort térmico }\end{array}$ & & & $\checkmark$ & \\
\hline & IEQc8.1 & Luz diurna y vistas - Luz diurna & & & $\checkmark$ & \\
\hline & IEQc8.2 & Luz diurna y vistas - Vistas & & & $\checkmark$ & \\
\hline $\begin{array}{l}\text { Innovación en el Diseño } \\
\text { (Innovation in Design) }\end{array}$ & IDc1 & Innovación en Diseño & & & & $\checkmark$ \\
\hline
\end{tabular}

Tabla 4. Créditos LEED con potencial de promover CPA en el ocupante. Fuente: Elaboración de los autores.

(*) Nota. Estos créditos pertenecen a una categoría distinta de los "Prerrequisitos" determinados por LEED, los cuales establecen los requisitos mínimos que todos los edificios deben cumplir para lograr la certificación LEED. Los primeros, en cambio, se definen como "los que realmente distinguen (al) edificio del resto" (USGBC, 2016).

sustentables presentes en el edificio, para instruir a futuros inquilinos y ocupantes sobre las estrategias sustentables del edificio. La categoría "Otros" se vio justificada por la revisión de la literatura y la validación de expertos, que apuntan a los créditos de innovación en el diseño, como oportunidades adicionales para promover sustentabilidad en los ocupantes.

A continuación, en la Tabla 4, se presentan los créditos LEED con potencial de promover CPA en el ocupante, según el Manual LEED-CS v2009 "Core and Shell" (v3), en su versión en inglés (USGBC, 2016), y traducidos para este trabajo al castellano.

Para obtener la información del uso de los créditos LEED-
CS v2009 "Core and Shell" en edificios de oficina de Argentina (AR), Chile (CL), Colombia (CO) y Perú (PE), con potencial de promover CPA, se vació la información de los puntajes de cada proyecto (Scorecards). La Tabla 5 ilustra el porcentaje del uso de cada crédito con potencial de promover CPA en los ocupantes en los cuatro países, como también el promedio de uso de cada crédito por países, para poder jerarquizarlos, y la desviación estándar de cada crédito, para compararlos.

Los créditos que obtuvieron los tres primeros lugares en el uso, según el promedio, fueron:

1) "SSc4.1: Acceso a Transporte Público de Calidad", con 99,34\%; 


\begin{tabular}{|c|c|c|c|c|c|c|c|}
\hline \multirow{2}{*}{$\begin{array}{c}\text { Categoría } \\
\text { Ambiental LEED }\end{array}$} & \multirow{2}{*}{$\begin{array}{c}\text { Código } \\
\text { Crédito LEED }\end{array}$} & \multicolumn{4}{|c|}{ PAÍs (\%) } & \multirow{2}{*}{$\begin{array}{l}\text { Promedio } \\
(\%)\end{array}$} & \multirow{2}{*}{$\begin{array}{l}\text { Desviaciór } \\
\text { estándar }\end{array}$} \\
\hline & & $A R$ & $C L$ & co & PE & & \\
\hline \multirow{7}{*}{$\begin{array}{c}\text { Parcelas } \\
\text { Sustentables } \\
\text { (Sustainable Sites) }\end{array}$} & SSc2 & 100,00 & 100,00 & 96,00 & 97,37 & 98,34 & 1,99 \\
\hline & $\mathrm{SSc} 4.1$ & 100,00 & 100,00 & 100,00 & 97,37 & 99,34 & 1,32 \\
\hline & $\mathrm{SSc} 4.2$ & 100,00 & 86,96 & 88,00 & 92,11 & 91,77 & 5,92 \\
\hline & $\mathrm{SSc} 4.3$ & 83,33 & 88,41 & 92,00 & 94,74 & 89,62 & 4,93 \\
\hline & SSc4.4 & 75,00 & 56,52 & 40,00 & 39,47 & 52,75 & 16,81 \\
\hline & SSc5.2 & 66,67 & 68,12 & 88,00 & 47,37 & 67,54 & 16,60 \\
\hline & SSc9 & 100,00 & 100,00 & 94,00 & 92,11 & 96,53 & 4,08 \\
\hline $\begin{array}{c}\text { Energía y } \\
\text { Atmósfera (Energy } \\
\text { and Atmosphere) }\end{array}$ & EAc3 & 91,67 & 14,49 & 50,00 & 21,05 & 44,30 & 35,15 \\
\hline \multirow{3}{*}{$\begin{array}{l}\text { Calidad Ambiental } \\
\text { Interior (Indoor } \\
\text { Environmental } \\
\text { Quality) }\end{array}$} & IEQc6 & 4,17 & 8,70 & 12,00 & 5,26 & 7,53 & 3,55 \\
\hline & IEQc8.1 & 8,33 & 59,42 & 18,00 & 39,47 & 31,31 & 22,82 \\
\hline & IEQc8.2 & 58,33 & 79,71 & 70,00 & 71,05 & 69,77 & 8,78 \\
\hline
\end{tabular}

Tabla 5. Resultado del análisis. Fuente: Elaboración de los autores.

2) "SSc2: Densidad del Entorno y Usos Diversos", con 98,34\%; y,

3) "SSc9: Pautas de diseño y construcción para inquilinos", con $96,53 \%$.

En los tres últimos lugares se ubicaron:

9) "EAc3: Puesta en servicio mejorada", con 44,30\%;

10) "IEQc8.1: Luz diurna y vistas - Luz diurna", con 31,31\%; y,

11) "IEQc6: Controlabilidad de los sistemas", con $7,53 \%$.

Con respecto al resultado del cálculo de la desviación estándar, los dos valores mayores se dieron en los siguientes créditos LEED:

- "IEQc8.1: Luz diurna y vistas - Luz diurna", con una desviación de 22,82 puntos. Argentina registró el menor promedio del uso de ese crédito (8,33\%) y Chile, el mayor $(59,43 \%)$.

- "EAc3: Puesta en servicio mejorada" con una desviación de 35,15 puntos. Chile registró aquí el menor promedio del uso de ese crédito (14,49\%) y Argentina, el mayor $(91,67 \%)$.

\section{DISCUSIÓN}

Los dos primeros créditos LEED en el uso de edificios de oficinas sustentables en Argentina, Chile, Colombia y Perú, entre los años 2012 y 2020, con potencial para promover CPA en ocupantes fueron "SSc4.1: Acceso a Transporte Público de Calidad" (99,34\%) y "SSc2: Densidad del Entorno y Usos Diversos" (98,34\%). Ambos buscan promover la reducción en el uso del vehículo, una solución de comportamiento pro-ambiental a escala urbana, de ciudad. El tercer lugar corresponde al crédito LEED, "SSc9: Pautas de diseño y construcción para inquilinos" (96,53\%), el cual tiene el propósito de educar a los inquilinos sobre la implementación de características de diseño y construcción sustentables en el edificio. Estas pautas de diseño y construcción persiguen apoyar a los inquilinos a diseñar y construir interiores sustentables y, además, a adoptar prácticas de construcción sustentables, las que optan por incluir al ocupante en el diseño de edificios sustentables, al referir a factores conductuales y culturales, cruciales para la sustentabilidad, y al promocionar el nivel de conciencia de todas las partes interesadas (Stakeholders) (clientes, diseñadores, contratistas, inquilinos y ocupantes) sobre conceptos de desarrollo sustentable y edificios sustentables (Zuo y Zhao, 2014). Este crédito da cuenta también de los nuevos retos de incluir al ocupante en la solución en el diseño de edificios sustentables, ya que, según Hoffman y Henn (2008), los nuevos obstáculos "ya no son principalmente tecnológicos y económicos. En cambio, son sociales y psicológicos" (p. 391). De la misma manera, se podría pronosticar un cambio en el paradigma de sistemas de certificación centrados en el ocupante, en concreto, de los sistemas IWBI WELL, Fitwel y Living Building Challenge (California Polytechnic State University, 2020). Adicionalmente, esta tendencia podría derivar del interés de los estudios que identifican créditos involucrados en diversas categorías de evaluación o dimensiones orientados a los ocupantes (Gou, 2019; Gou, Prasad y SiuYu Lau, 2013; Illankoon, Tam, Le y Shen, 2017), o bien, como sugieren Wen et al., (2020), del aumento considerable que, en las últimas tres décadas, está adquiriendo el peso de la categoría social en los sistemas analizados. Junto a ello, 
se ha advertido un pequeño incremento en el peso de la categoría económica y una disminución continua en el de la categoría ambiental. Cabe agregar, siguiendo a Xue, Lau, Gou, Song y Jiang, (2019), que el diseño de edificios con sistemas de certificación debería pasar del enfoque de ingeniería centrado en la construcción, a un enfoque biofílico centrado en el ser humano.

El crédito LEED menos empleado en los cuatro países resultó ser el "IEQc6: Controlabilidad de los sistemas" (7,53\%). Dicha cifra puede asociarse con la intención de no incluir al ocupante en el diseño arquitectónico para cumplir con los objetivos de la sustentabilidad en el edificio, al minimizarse el impacto de la interacción del ocupante con el edificio a través del control personal. Dos son los términos ligados a esta tendencia, el "Rebound effect" (Efecto rebote) (Frondel, 2004; Grossmann, Galvin, Weiss, Madlener y Hirschl, 2016; Sorrell, 2007) y el "Robust design" (Diseño robusto) (Buso et al., 2015; Karjalainen, 2016; O'brien, 2013; Palme et al., 2006). Investigaciones vinculadas a la interacción del ocupante con el edificio a través del control personal y otros tópicos, como el confort térmico en el efecto en el ahorro de la energía (Nagy, Yong, Frei y Schlueter, 2015; Wagner, Gossauer, Moosmann, Gropp y Leonhart, 2007) y en la productividad en entornos laborales (Leaman y Bordass, 2001), constataron que la relación de un mayor control individual directo, conduce a un mayor confort térmico (De Dear y Brager, 2002; Karjalainen y Koistinen, 2007) y a una mayor satisfacción (Brager y Baker, 2009; Fountain, Brager y de Dear, 1996). Desde un punto de vista psicológico, otros estudios observaron que el control personal es un factor importante para aumentar la satisfacción y la productividad del ocupante (Samani, 2015; Vine, Lee, Clear, DiBartolomeo y Selkowitz, 1998).

Con respecto a las cifras arrojadas tras el cálculo de la desviación estándar -35,15 del crédito LEED "EAc3: Puesta en servicio mejorada" y 22,82 de "IEQc8.1: Luz diurna y vistas - Luz diurna"- las cuales dieron evidencia de la dispersión de los valores entre los casos de Argentina y Chile, se puede indicar que conocer las posibles causas de ello representa una valiosa oportunidad de investigación. Desde esa perspectiva, como trabajos futuros, se prevé analizar los casos de Brasil y México, por ser los países con mayor cantidad de edificios certificados LEED de la región, pero asimismo el sistema local de Chile, CES. La metodología propuesta puede replicarse en ambos estudios, y los resultados pueden, por lo tanto, compararse.

\section{CONCLUSIÓN}

En la relación del edificio sustentable y el ocupante, en el marco del diseño de edificios de oficinas que procuran lograr objetivos de sustentabilidad, los créditos más empleados en los países seleccionados optan por incluir al ocupante en el uso y la promoción de transportes alternativos, lo cual contribuye a reducir el impacto en el ambiente a nivel urbano; una solución que apoyarían los tres grupos propuestos en
Hopwood, Mellor y O'Brien (2005) para enfrentar los desafíos del desarrollo sustentable. Otra alternativa para incorporar al ocupante en el diseño, referida en el crédito LEED que busca instruir al ocupante en las estrategias sustentables presentes en el edificio, también interviene en la sociedad, pero a través de la cultura; término que se asocia con la acción de cultivar o practicar algo. Y una última solución, que busca restringir la interacción del ocupante en el edificio, con el objetivo de lograr una eficiencia en el uso de la energía (como medida de sustentabilidad), aporta a la sociedad, esta vez, mediante la tecnología, es decir, de la aplicación de la ciencia a la resolución de problemas concretos.

Finalmente, hay que señalar que identificar criterios, a través de los créditos en un sistema de certificación para promover sustentabilidad en los ocupantes —en los CPA-, va en sintonía con el surgmiento de nuevos sistemas de certificación enfocados en la sustentabilidad social, hacia el ocupante, en vez de hacerlo hacia la sustentabilidad ambiental, la eficiencia energética y el uso de las tecnologías en los edificios. Por ello, precisamente, es que el presente trabajo representa un aporte en el fenómeno de la relación de los edificios sustentables y el ocupante.

\section{AGRADECIMIENTOS}

La tesis de doctorado, en la cual se basa este artículo, fue posible gracias a la Beca DAU (Doctorado en Arquitectura y Urbanismo) - Dirección de Postgrados UBB (Universidad del Bío-Bío) y al proyecto Fondecyt Regular No 1201456 "Criterios innovadores de bienestar para el diseño y evaluación de edificios sustentables desde la perspectiva de los ocupantes".

\section{REFERENCIAS BIBLIOGRÁFICAS}

Ahmed, M., Abul Hasan, M. y Mallick, J. (2016). World Green Building Rating Systems: A Comparative Study. En International Conference Cum Exhibition On Building Utilities 2016. Recuperado de https://www.researchgate.net/publication/323771840

Ajzen, I. (1991). The Theory of Planned Behavior. Organizational Behavior and Human Decision Processes, 50(2), 179-211. DOl: https://doi.org/https://doi.org/10.1016/0749-5978(91)90020-T

Bamberg, S. y Möser, G. (2007). Twenty years after Hines, Hungerford, and Tomera: A new meta-analysis of psychosocial determinants of pro-environmental behaviour. Journal of Environmental Psychology, 27(1), 14-25. DOI: https://doi. org/10.1016/j.jenvp.2006.12.002

Bolton, L. E., Cohen, J. B. y Bloom, P. N. (2006). Does Marketing Products as Remedies Create "Get Out of Jail Free Cards"? Journal of Consumer Research, 33(1), 71-81. https://doi. org/10.1086/504137

Brager, G. y Baker, L. (2009). Occupant satisfaction in mixed-mode buildings. Building Research and Information, 37(4), 369-380. DOI: https://doi.org/10.1080/09613210902899785 
Browne y Frame, I. (1999). Green buildings need green occupants. Eco-Management and Auditing, 6(2), 80-85. DOI: https://doi.org/10.1002/(sici)1099-0925(199906)6:2<80::aidema102>3.0.co;2-\#

Buso, T., Fabi, V., Andersen, R. K. y Corgnati, S. (2015). Occupant behaviour and robustness of building design. Building and Environment, 94, 694-703. DOI: https://doi. org/10.1016/j.buildenv.2015.11.003

Catlin, J. R. y Wang, Y. (2013). Recycling gone bad: When the option to recycle increases resource consumption. Journal of Consumer Psychology, 23(1), 122-127. DOI: https://doi. org/10.1016/j.jcps.2012.04.001

Chile GBC (2020). Reporte Construccion Sustentable Chile GBC - Diciembre 2020. Recuperado de www.chilegbc.cl

Chwieduk, D. (2003). Towards sustainable-energy buildings. Applied Energy, 76(1-3), 211-217. DOI: https://doi. org/10.1016/S0306-2619(03)00059-X

Cole, L. B. (2013). The Green Building as a Medium for Environmental Education. Michigan Journal of Sustainability, 1. DOI: https://doi.org/10.3998/mjs.12333712.0001.012

Cole, L. B. (2014). The Teaching Green School Building: a framework for linking architecture and environmental education. Environmental Education Research, 20(6), 836857. DOI: https://doi.org/10.1080/13504622.2013.833586

Cole, L. B. (2018). The Teaching Green Building: Five Theoretical Perspectives. En W. Leal Filho, R. W. Marans y J. Callewaert (Eds.), Handbook of Sustainability and Social Science Research (pp. 107-125). Springer International Publishing. DOI: https://doi.org/10.1007/978-3-319-671222_6

Cole, L. B. y Hamilton, E. M. (2019). Can a green school building teach? A pre- and post-occupancy evaluation of a teaching green school building. Environment and Behavior, 52(10), 1047-1078. DOI: https://doi.org/10.1177/0013916518825283

Cole, R. J. (1999). Building environmental assessment methods: Clarifying intentions. Building Research and Information, 27(45), 230-246. DOI: https://doi.org/10.1080/096132199369354

Cole, R. J. (2002). Emerging trends in building environmental assessment methods. Building Research \& Information, 26(1), 3-16. DOI: https://doi.org/10.1080/096132198370065

Cranz, G., Lindsay, G., Morhayim, L. y Lin, A. (2014). Communicating Sustainability: A Postoccupancy Evaluation of the David Brower Center. Environment and Behavior, 46(7), 826-847. DOI: https://doi.org/10.1177/0013916513475449

D'Oca, S., Hong, T. y Langevin, J. (2018). The human dimensions of energy use in buildings: A review. Renewable and Sustainable Energy Reviews, 81, pp. 731-742. DOI: https://doi.org/10.1016/j.rser.2017.08.019

De Dear, R. J. y Brager, G. (2002). Thermal comfort in naturally ventilated buildings: Revisions to ASHRAE Standard 55. Energy and Buildings, 34(6), 549-561. DOI: https://doi. org/10.1016/S0378-7788(02)00005-1
Deuble, M. P. (2007). Green Occupants for Green Buildings. Macquarie.

Deuble, M. P. y de Dear, R. J. (2012). Green occupants for green buildings: The missing link? Building and Environment, 56(56), 21-27. DOI: https://doi.org/10.1016/j.buildenv.2012.02.029

Deuble, M. P. y de Dear, R. J. (2009). Do green buildings need green occupants? En Proceedings of Healthy Buildings 2009 (pp. 843-846). Recuperado de https://researchers.mq.edu.au/en/ publications/do-green-buildings-need-green-occupants

Ding, G. K. C. C. (2008). Sustainable construction-The role of environmental assessment tools. Journal of Environmental Management, 86(3), 451-464. DOI: https://doi.org/10.1016/j. jenvman.2006.12.025

Dunlap, R. E. y Van Liere, K. D. (1978). The "new environmental paradigm". Journal of Environmental Education, 9(4), 10-19. DOI: https://doi.org/10.1080/00958964.1978.10801875

Dunlap, R. E., Van Liere, K. D., Mertig, A. G. y Jones, R. E. (2000). New Trends in Measuring Environmental Attitudes: Measuring Endorsement of the New Ecological Paradigm: A Revised NEP Scale. Journal of Social Issues, 56(3), 425-442. DOI: https://doi. org/10.1111/0022-4537.00176

Fabi, V., Andersen, R. y Corgnati, S. (2011). Description of occupant behaviour in building energy simulation: state-of-art and concepts for improvements. Building Simulation 2011: 12th Conference of International Building Performance Simulation Association, Sydney, 14-16 November, 2882-2889.

Fountain, M., Brager, G. y de Dear, R. (1996). E IEI GY AHD BUILDIPIG Expectations of indoor climate control. Energy and Buildings, 24(3), 179-182

Frondel, M. (2004). Guest editorial: Energy conservation, the rebound effect, and future energy and transport technologies: An introduction to energy conservation and the rebound effect. International Journal of Energy Technology and Policy, 2(3), 203208.

Garrote, P. R. y Rojas, M. del C. (2015). La validación por juicio de expertos: dos investigaciones cualitativas en Lingüística aplicada. Revista Nebrija de Lingüística Aplicada a la Enseñanza de Lenguas, 18, 124-139.

Gou, Z. (2016). Green building for office interiors: challenges and opportunities. Facilities, 34(11-12), 614-629. DOI: https://doi. org/10.1108/F-04-2015-0022

Gou, Z. (2019). Human Factors in Green Building: Building Types and Users' Needs. Buildings, 9(1), 17. DOI: https://doi. org/10.3390/buildings 9010017

Gou, Z., Prasad, D. y Siu-Yu Lau, S. (2013). Are green buildings more satisfactory and comfortable? Habitat International, 39, 156-161. DOI: https://doi.org/10.1016/j.habitatint.2012.12.007

Grossmann, D., Galvin, R., Weiss, J., Madlener, R. y Hirschl, B. (2016). A methodology for estimating rebound effects in nonresidential public service buildings: Case study of four buildings in Germany. Energy and Buildings, 111, 455-467 DOI: https://doi. org/10.1016/j.enbuild.2015.11.063 
Harland, P., Staats, H. y Wilke, H. A. M. (1999). Explaining Proenvironmental Intention and Behavior by Personal Norms and the Theory of Planned Behavior. Journal of applied social psychology, 29(12), 2505-2528. DOI: https://doi. org/10.1111/j.1559-1816.1999.tb00123.x

Heerwagen, J. (2000). Green buildings, organizational success and occupant productivity. Building Research and Information, 28(56), 353-367. DOI: https://doi.org/10.1080/096132100418500

Hernández Sampieri, R., Fernández Collado, C. y del Pilar Baptista Lucio, M. (2010). Metodología de la Investigación. México D.F: McGraw Hill.

Hill, A., Han, Y., Taylor, J. E., Shealy, T., Pearce, A. y Mohammadi, N. (2019). Empirical examination of pro-environmental behaviors in traditional, green featured and LEED certified buildings. Energy Procedia, 158, 3982-3987. DOI: https://doi.org/10.1016/j. egypro.2019.01.843

Hoffman, A. y Henn, R. (2008). Overcoming the Social and Psychological Barriers to Green Building. Organization \& Environment, 21(4), 390-419. DOl: https://doi. org/10.1177/1086026608326129

Hong, T., Yan, D., D’Oca, S. y Chen, C. (2017). Ten questions concerning occupant behavior in buildings: The big picture. Building and Environment, 114, 518-530. DOI: https://doi. org/10.1016/j.buildenv.2016.12.006

Hopwood, B., Mellor, M. y O’Brien, G. (2005). Sustainable development: Mapping different approaches. Sustainable Development, 13(1), 38-52. DOI: https://doi.org/10.1002/sd.244

Hostetler, M. y Noiseux, K. (2010). Are green residential developments attracting environmentally savvy homeowners? Landscape and Urban Planning, 94(3-4), 234-243. DOI: https:// doi.org/10.1016/j.landurbplan.2009.10.008

Illankoon, I. M. C. S., Tam, V. W. Y., Le, K. N. y Shen, L. (2017). Key credit criteria among international green building rating tools. Journal of Cleaner Production, 164, 209-220. DOl: https://doi. org/10.1016/j.jclepro.2017.06.206

Karjalainen, S. (2016). Should we design buildings that are less sensitive to occupant behaviour? A simulation study of effects of behaviour and design on office energy consumption. Energy Efficiency, 9(6), 1257-1270. DOI: https://doi.org/10.1007/s12053015-9422-7

Karjalainen, S. y Koistinen, O. (2007). User problems with individual temperature control in offices. Building and Environment, 42(8), 2880-2887. DOI: https://doi.org/10.1016/j.buildenv.2006.10.031

Khashe, S., Heydarian, A., Gerber, D., Becerik-Gerber, B., Hayes, T. y Wood, W. (2015). Influence of LEED branding on building occupants' pro-environmental behavior. Building and Environment, 94, 477-488. DOI: https://doi.org/10.1016/j. buildenv.2015.10.005

Kirk, J. L. (2010). Sustainable environments and pro-environmental behavior. University of Nebraska at Lincoln. Recuperado de http:// digitalcommons.unl.edu/arch_id_theseshttp://digitalcommons. unl.edu/arch_id_theses/2
Kollmuss, A. y Agyeman, J. (2002). Mind the Gap: Why do people act environmentally and what are the barriers to pro-environmental behavior? Environmental Education Research, 8(3), 239-260. DOI: https://doi.org/10.1080/13504620220145401

Labartino, I. (2018). Building certification as a driver in green building design: The holistic apporach of WELL. Stockholm: KTH, School of Architecture and the Built Environment.

Leaman, A. y Bordass, B. (2001). Assessing building performance in use 4: The Probe occupant surveys and their implications. Building Research and Information, 29(2), 129-143. DOI: https:// doi.org/10.1080/09613210010008045

Lee, Y. S. (2010). Office layout affecting privacy, interaction, and acoustic quality in LEED-certified buildings. Building and Environment, 45(7), 1594-1600. DOI: https://doi.org/10.1016/j. buildenv.2010.01.007

Lindenberg, S. y Steg, L. (2007). Normative, Gain and Hedonic Goal Frames Guiding Environmental Behavior. Journal of Social Issues, 63 (117-137). DOI: 10.1111/j.1540-4560.2007.00499.x

Mattinzioli, T., Sol-Sánchez, M., Moreno, B., Alegre, J. y Martínez, G. (2020). Sustainable building rating systems: A critical review for achieving a common consensus. Critical Reviews in Environmental Science and Technology, 51(5), 512-534. DOI: https://doi.org/10 .1080/10643389.2020.1732781

McCunn, L. J. y Gifford, R. (2012). Do green offices affect employee engagement and environmental attitudes? In Architectural Science Review, 55(2), 128-134. DOI: https://doi.org/10.1080/0 0038628.2012 .667939

Moezzi, M. (2009). Are comfort expectations of building occupants too high? Building Research and Information, 37(1), 79-83. DOI: https://doi.org/10.1080/09613210802611009

Mokhtar, N. S. y Wilkinson, S. (2015). Motivation Factors in Energy Saving Behaviour between Occupants in Green and Conventional Buildings - Malaysia Case Study. International Journal of Environmental Science and Development, 6(7), 491-497. DOI: https://doi.org/10.7763/ijesd.2015.v6.643

Mokhtar, N. S., Wilkinson, S. y Fassman, E. (2015). Strategies for improving energy saving behaviour in commercial buildings in Malaysia. Engineering, Construction and Architectural Management, 22(1), 73-90. DOI: https://doi.org/10.1108/ECAM04-2014-0054

Nagy, Z., Yong, F. Y., Frei, M. y Schlueter, A. (2015). Occupant centered lighting control for comfort and energy efficient building operation. Energy and Buildings, 94, 100-108. DOI: https://doi. org/10.1016/j.enbuild.2015.02.053

O'brien, W. (2013). Occupant-proof buildings: can we design buildings that are robust against occupant behaviour. 13th Conference of International Building Performance Simulation Association, Chambéry, France, August 26-28 (pp. 746-1754).

Palme, M., Isalgue, A., Coch-Roura, H., Serra, R. y Coch, H. (2006). Robust design: A way to control energy use from the human behaviour in architectural spaces. En PLEA 2006 - 23rd International Conference on Passive and Low Energy Architecture, Conference Proceedings, September (pp. |311-1316). 
Pierson, L. (2020). Building for Wellness-Healthy Building Course for Cal Poly SLO CM Department. Recuperado de https:// digitalcommons.calpoly.edu/cmsp/365/

Ramkissoon, H., Weiler, B. y Smith, L. D. G. (2012). Place attachment and pro-environmental behaviour in national parks: The development of a conceptual framework. Journal of Sustainable Tourism, 20(2), 257-276. DOI: https://doi.org/10.108 $0 / 09669582.2011 .602194$

Rashid, M., Spreckelmeyer, K., \& Angrisano, N. J. (2012). Green buildings, environmental awareness, and organizational image. Journal of Corporate Real Estate, 14(1), 21-49. DOI: https://doi. org/10.1108/14630011211231428

Samani, S. A. (2015). The impact of personal control over office workspace on environmental satisfaction and performance. Journal of Social Sciences and Humanities, 1(3), 163-175. Recuperado de http://www.aiscience.org/journal/jssh

Sorrell, S. (2007). The rebound effect: an assessment of the evidence for economy-wide energy savings from improved energy efficiency. Australian family physician, 42(4). UK Energy Research Centre. DOI: https://doi.org/1-903144-0-35

Stazi, F. y Naspi, F. (2018). Impact of Occupants' Behaviour on Zero-Energy Buildings. Cham: SpringerBriefs in Energy.

Steinberg, D., Patchan, M., Schunn, C. y Landis, A. (2009). Determining Adequate Information for Green Building Occupant Training Materials. Journal of Green Building, 4(3), 143-150.

Stern, P. C. (2000). Toward a Coherent Theory of Environmentally Significant Behavior. Journal of Social Issues, 56(3), 407-424.

Tezel, E. y Giritli, H. (2019). Understanding pro-environmental workplace behavior: a comparative study. Facilities, 37(9-10), 669-683. DOI: https://doi.org/10.1108/F-12-2017-0134

USGBC (2016). LEED 2009 for Core and Shell Development Rating System. Recuperado de https://www.usgbc.org/resources/ leed-2009-core-and-shell-current-version

Van den Brom, P., Meijer, A. y Visscher, H. (2016). Reducing the performance gap by analysing specific combinations of occupant and building characteristics. En 4th European Conference on Behaviour and Energy Efficiency (Behave 2016), September, 8-9. Recuperado de http://pure.tudelft.nl/ws/files/10412927/Brom_ Meijer_Visscher.pdf

Vine, E., Lee, E., Clear, R., DiBartolomeo, D. y Selkowitz, S. (1998). Office worker response to an automated venetian blind and electric lighting system: a pilot study. Energy and Buildings, 28(2), 205-218.

Wagner, A., Gossauer, E., Moosmann, C., Gropp, T. y Leonhart, R. (2007). Thermal comfort and workplace occupant satisfactionResults of field studies in German low energy office buildings. Energy and Buildings, 39(7), 758-769. DOI: https://doi. org/10.1016/j.enbuild.2007.02.013

Wen, B., Musa, N., Onn, C. C., Ramesh, S., Liang, L. y Wang, W. (2020). Evolution of sustainability in global green building rating tools. Journal of Cleaner Production, 259. DOI: https://doi. org/10.1016/j.jclepro.2020.120912
Wu, S. R. (2016). Green buildings and green users: an assessment of using green building environments to communicate sustainability to users. Michigan: Michigan State.

Wu, S. R., Greaves, M., Chen, J. y Grady, S. C. (2017). Green buildings need green occupants: a research framework through the lens of the Theory of Planned Behaviour. Architectural Science Review, 60(1), 5-14. https://doi.org/10.1080/00038628.2016.119 7097

Wu, S. R., Green, M., Chen, J., Tang, Y. y Yang, A. (2015). Green building design and visual persuasion on occupants' pro environmental behaviours. Living and Learning: Research for a Better Built Environment, 49th International Conference of the Architectural Science Association, 133-142.

Wu, S. R., Kim, S.-K., Park, H., Fan, P., Ligmann-Zielinska, A. y Chen, J. (2017). How do green buildings communicate green design to building users? A survey study of a leed-certified building. Journal of Green Building, 12(3), 85-100. DOI: https:// doi.org/doi.org/10.3992/1943-4618.12.3.85

Xue, F., Lau, S. S. Y., Gou, Z., Song, Y. y Jiang, B. (2019). Incorporating biophilia into green building rating tools for promoting health and wellbeing. Environmental Impact Assessment Review, 76, 98-112. DOI: https://doi.org/10.1016/j.eiar.2019.02.004

Zuo, J. y Zhao, Z. Y. (2014). Green building research-current status and future agenda: A review. Renewable and Sustainable Energy Reviews, 30, 271-281. DOI: https://doi.org/10.1016/j. rser.2013.10.021 